\title{
Genome-Wide Analysis of Group A Streptococci Reveals a Mutation That Modulates Global Phenotype and Disease Specificity
}

\author{
Paul Sumby ${ }^{1,2}$, Adeline R. Whitney ${ }^{3}$, Edward A. Graviss ${ }^{2}$, Frank R. DeLeo ${ }^{3}$, James M. Musser ${ }^{1,2^{*}}$ \\ 1 Center for Molecular and Translational Human Infectious Diseases Research, The Methodist Hospital Research Institute, Houston, Texas, United States of America, 2 Center \\ for Human Bacterial Pathogenesis Research, Department of Pathology, Baylor College of Medicine, Houston, Texas, United States of America, $\mathbf{3}$ Laboratory of Human Bacterial \\ Pathogenesis, Rocky Mountain Laboratories, National Institute of Allergy and Infectious Diseases, National Institutes of Health, Hamilton, Montana, United States of America
}

Many human pathogens produce phenotypic variants as a means to circumvent the host immune system and enhance survival and, as a potential consequence, exhibit increased virulence. For example, it has been known for almost 90 y that clinical isolates of the human bacterial pathogen group A streptococci (GAS) have extensive phenotypic heterogeneity linked to variation in virulence. However, the complete underlying molecular mechanism(s) have not been defined. Expression microarray analysis of nine clinical isolates identified two fundamentally different transcriptomes, designated pharyngeal transcriptome profile (PTP) and invasive transcriptome profile (ITP). PTP and ITP GAS differed in approximately $10 \%$ of the transcriptome, including at least 23 proven or putative virulence factor genes. ITP organisms were recovered from skin lesions of mice infected subcutaneously with PTP GAS and were significantly more able to survive phagocytosis and killing by human polymorphonuclear leukocytes. Complete genome resequencing of a mouse-derived ITP GAS revealed that the organism differed from its precursor by only a 7bp frameshift mutation in the gene (covS) encoding the sensor kinase component of a two-component signal transduction system implicated in virulence. Genetic complementation, and sequence analysis of covR/S in 42 GAS isolates confirmed the central role of covR/S in transcriptome, exoproteome, and virulence modulation. Genome-wide analysis provides a heretofore unattained understanding of phenotypic variation and disease specificity in microbial pathogens, resulting in new avenues for vaccine and therapeutics research.

Citation: Sumby P, Whitney AR, Graviss EA, DeLeo FR, Musser JM (2006) Genome-wide analysis of group A streptococci reveals a mutation that modulates global phenotype and disease specificity. PLoS Pathog 2(1): e5.

\section{Introduction}

Bacterial pathogens have long been recognized to undergo phenotypic variation (reviewed in [1]). Historically, interest in this phenomenon has been fueled by the observation that phenotypic variants can differ in pathogenesis characteristics, such as increased or decreased virulence, or adaptation to a particular anatomic site. Extensive work has been directed at elucidating the molecular genetic events that contribute to phenotypic variation, with antigenic variation being the best-studied category. With few exceptions, most studies have focused on analysis of a distinct phenotype such as adhesin production or lipooligosaccharide structural modification. Several molecular mechanisms have been documented to contribute to phenotypic variation, the most common being slipped-strand mispairing events that result in phase-variable expression of the associated gene [1].

The group A streptococci (GAS) cause many distinct human infections [2]. Disease manifestations range from mild infections such as pharyngitis ("strep throat") and impetigo, to extensive tissue destruction in the case of necrotizing fasciitis (the "flesh-eating" syndrome). Postinfection sequelae such as rheumatic fever and glomerulonephritis can also occur. The mechanisms that enable GAS to cause diverse diseases are unknown, although both bacterial and hostspecific components are thought to be involved [3].

Associated morphologic and virulence variation in GAS has been known for almost 90 y [4,5]. Classic studies identified GAS phenotypic variation during invasive and upper respi- ratory tract infections $[4,6]$. More recently, correlations have been reported between the source of GAS clinical isolates and their ability to invade human epithelial cells or secrete high concentrations of virulence factors such as streptococcal pyrogenic exotoxin A, B, and C (SpeA, SpeB, and SpeC), or streptolysin O (SLO) [7-9]. Such correlations have been observed for multiple GAS serotypes, including clonal contemporary serotype M1 GAS [10]. The idea that GAS phenotypic heterogeneity contributes to distinct disease manifestations is supported by the identification of inherited alterations in virulence factor production when GAS is passaged in human blood ex vivo or through mice [5,11-14].

Virulence factor production by GAS is regulated by standalone transcription factors and two-component signal trans-

Editor: Partho Ghosh, University of California San Diego, United States of America Received October 19, 2005; Accepted December 20, 2005; Published January 27, 2006

\section{DOI: 10.1371/journal.ppat.0020005}

This is an open-access article distributed under the terms of the Creative Commons Public Domain declaration which stipulates that, once placed in the public domain, this work may be freely reproduced, distributed, transmitted, modified, built upon, or otherwise used by anyone for any lawful purpose.

Abbreviations: cov, control of virulence; GAS, group A streptococci; ITP, invasive transcriptome profile; PMN, polymorphonuclear leukocyte; PTP, pharyngeal transcriptome profile; SLO, streptolysin O; Spd3, Streptococcus pyogenes DNase 3; Spe, streptococcal pyrogenic exotoxin; SPN, Streptococcus pyogenes NAD-glycohydrolase; TCS, two-component signal transduction system; THY, Todd-Hewitt broth with yeast extract

* To whom correspondence should be addressed. E-mail: jmmusser@tmh.tmc.edu 


\section{Synopsis}

Phenotypic heterogeneity within an infecting population is a strategy commonly used by bacterial pathogens to evade the host immune system and enhance survival. Such phenotypic variation has been observed for the human pathogen group A streptococci (GAS), which can cause a wide range of diseases with differing severity. However, the underlying mechanisms that control this variation, and the survival- and virulence-associated effects of this variation, have not been fully elucidated.

By assaying total gene expression the authors found that clinical GAS isolates from invasive and pharyngeal diseases had distinct gene expression patterns during growth in standard laboratory media. These two gene expression patterns conferred distinct virulence-associated attributes on the expressing GAS strain, as assessed using bacteremia and soft-tissue infection models of disease. Likewise, the ability to survive the bactericidal activity of human neutrophils was significantly different between GAS strains with the two distinct expression patterns. Transition from one gene expression pattern to the other required the mutation of the twocomponent signal transduction system CovRS (control of virulence $\mathrm{R} / \mathrm{S}$ ). The authors conclude that the ability of GAS to remodel its transcriptome plays a major contribution in its ability to colonize distinct niches of the human body and cause disease.

duction systems (TCSs) [15]. Thirteen TCSs have been described in GAS, of which the CovRS system (also known as CsrRS) is the best characterized. CovRS is a negative regulatory TCS that directly or indirectly influences expression of $10 \%$ to $15 \%$ of GAS genes, including several virulence factors [16-21].

Despite these advances, we have an imprecise understanding of the contribution of phenotypic variation to hostpathogen interactions in GAS, and the molecular mechanism(s) controlling this heterogeneity. Recently, genome-wide investigative strategies have been used successfully to provide new information about GAS population genetics, evolution, and pathogenesis [22]. Inasmuch as phenotypic variation in GAS may be a key component of the pathogen life cycle, we chose to investigate this phenomenon using genome-wide analytic strategies, including transcriptome profiling and genome resequencing. Here we report genome, transcriptome, and partial secretome differences that distinguish GAS isolated from invasive and pharyngeal infections and permit a heretofore unattainable understanding of phenotypic variation in a microbial pathogen.

\section{Results}

Transcriptome-Based Grouping of Serotype M1 GAS Strains

The transcriptomes of nine contemporary (post-1987) serotype M1 GAS strains grown to early exponential phase in Todd-Hewitt broth with yeast extract (THY) were analyzed with an Affymetrix expression microarray. These nine strains included six from patients with pharyngitis and three from invasive disease episodes and were selected from approximately 2,000 genetically characterized serotype M1 strains [10]. Two very distinct transcriptome clusters were identified based on analysis of the microarray data (Figure 1A). The three invasive isolates formed one cluster termed an invasive transcriptome profile (ITP), and the six pharyngitis isolates formed a second cluster termed a pharyngeal transcriptome profile (PTP).
The data imply that GAS strains cultured from patients with pharyngeal and invasive disease have distinct transcriptomes, which are retained upon in vitro growth. Analysis of differential gene expression between the two transcriptome profiles identified 89 genes that were statistically significant ( $t$-test followed by a false discovery rate correction, $Q<0.05$ ) and transcribed 2-fold or greater between the two profiles (increasing to 176 genes at a 1.5-fold change). The mean fold change in transcripts of 24 selected virulence factors or virulence factor regulators between the three ITP and six PTP strains are shown in Figure 1B. How the transcriptional variation of these 24 virulence factors/ regulators relate in context to the global transcriptional variation occurring between the three ITP and six PTP strains is shown in Figure $\mathrm{S} 1$ (see Supporting Information). Differential transcription of virulence factors between ITP
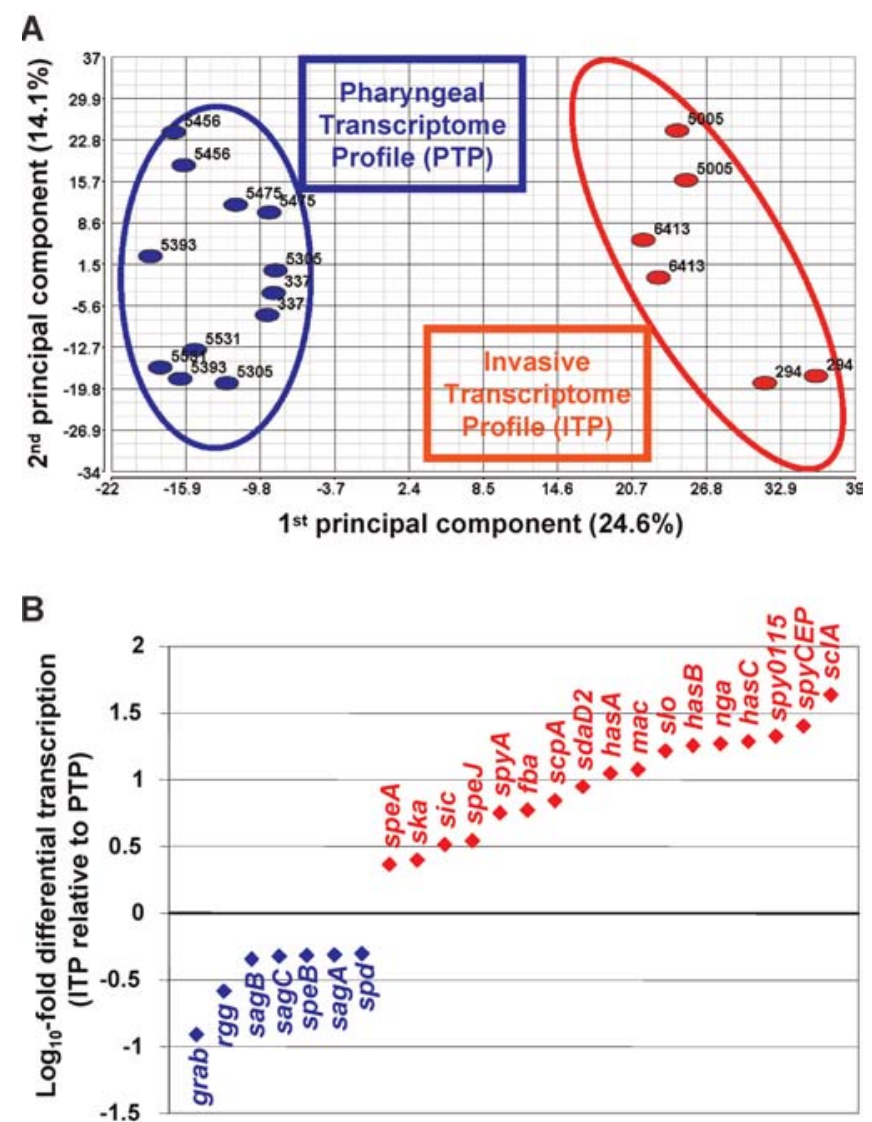

Figure 1. Differential Gene Expression of ITP and PTP GAS

(A) Principal component analysis plot showing transcriptome differences between invasive (red) and pharyngeal (blue) isolates. Principal component analysis assesses the variance in a dataset in terms of principal components. The two most significant principal components are displayed on the $\mathrm{x}$ - and $\mathrm{y}$-axes. Ellipses (calculated using $2 \mathrm{X}$ standard deviation for each gene per group) have been superimposed to highlight group differences between ITP and PTP strains. Numbered data points refer to the MGAS strain number from which the RNA sample was isolated. The percentages of the total variation that are accounted for by the 1st and 2nd principal components are shown on the $x$ - and $y$-axes labels.

(B) $\log _{10}$-fold transcript differences between the three ITP and six PTP isolates shown in (A) for select virulence genes and virulence gene regulators. Genes that are expressed higher in ITP isolates are shown in red; those expressed higher in PTP isolates are shown in blue. All genes shown are statistically significant ( $t$-test followed by a false discovery rate correction, $Q<0.05$ ).

DOI: 10.1371/journal.ppat.0020005.g001 
A
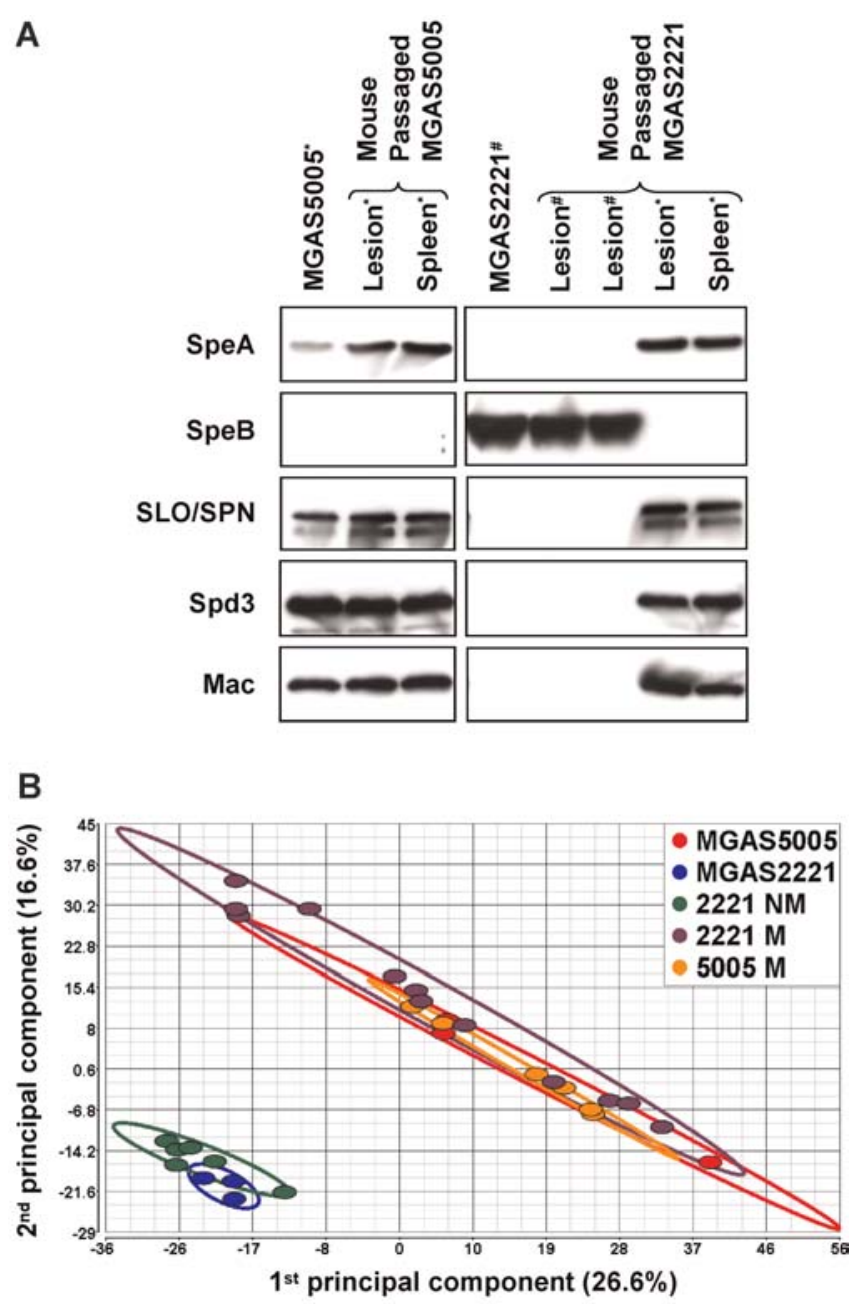

C

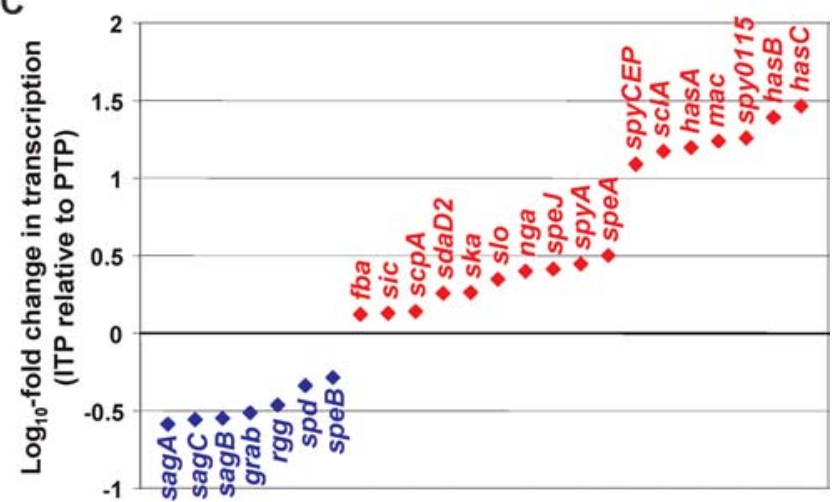

Figure 2. Mouse Passage Results in Recovery of ITP Derivatives from Infecting PTP GAS

(A) Western immunoblots of supernatant proteins obtained from overnight cultures of ITP and PTP GAS isolates, before and after mouse passage. The amount of immunoreactive SpeA, SpeB, SLO/SPN, Spd3, and Mac was unaffected by mouse passage of the ITP strain MGAS5005, whereas they were affected by all spleen and some lesion-isolated GAS after mouse passage of the PTP GAS isolate MGAS2221. Strains with a mucoid colony morphology are indicated with an asterisk (*), while nonmucoid strains are indicated by a hash (\#).

(B) Principal component analysis plot portraying PTP to ITP transition for several mouse-passaged derivatives of strain MGAS2221. The 2221 NM (green) and $2221 \mathrm{M}$ (purple) data points are mouse-passaged derivatives of strain MGAS2221 with distinct nonmucoid and mucoid colony morphologies, respectively. The $5005 \mathrm{M}$ (orange) data points are mouse-passaged derivatives of strain MGAS5005 that retain the mucoid colony morphology of the parental strain. Ellipses (calculated using $2 \mathrm{X}$ standard deviation for each gene per group) have been superimposed to highlight differences between ITP and PTP GAS. The percentages of the total variation that are accounted for by the 1 st and 2 nd principal components are shown on the $x$ - and $y$-axes labels.

(C) $\log _{10}$-fold differences in transcripts for select virulence genes and virulence gene regulators between all the PTP and ITP strains shown in (B). Genes expressed higher in ITP derivatives are shown in red; those expressed higher in PTP derivatives are shown in blue. RNA was isolated from bacteria grown to the exponential phase of growth in THY media. All genes shown are statistically significant as assessed by a $t$-test followed by a false discovery rate correction of $Q<0.05$.

DOI: 10.1371/journal.ppat.0020005.g002

and PTP isolates raises the possibility that certain GAS virulence factors have a disease-specific function.

\section{ITP GAS Are Derived from PTP GAS during Invasive Infections}

Inasmuch as the ITP GAS were isolated from cases of invasive disease, we hypothesized that ITP GAS may be derived from PTP GAS during invasive infections. To test this hypothesis, GAS were isolated from the spleens and skin lesions of mice euthanized $5 \mathrm{~d}$ after subcutaneous injection with strain MGAS2221 (a representative PTP strain) or strain MGAS5005 (a representative ITP strain) (Figure S2). A total of approximately 1,000 colonies obtained from infected mice were inspected visually for colony morphology. Colony morphology was used as an initial indicator of transcriptional profile because PTP GAS are nonmucoid, whereas ITP GAS are mucoid. This difference in colony morphology correlates with the increased expression of the capsule synthesis operon (has $A B C$ ) by ITP isolates (Figure 1B). Regardless of the colony morphology of the original infecting strain, all GAS isolated from the spleens of infected animals were mucoid. These data imply that our hypothesis that PTP GAS have the ability to transition to ITP GAS during invasive infections was correct. More important, although all GAS isolated from the skin lesions of mice infected with the ITP strain also were mucoid, colonies grown from the skin lesions of PTP-infected mice were a mixture of nonmucoid parental and mucoid variant strains at approximately 1:1 ratio (Figure S2).

To provide additional evidence that the mucoid derivatives of strain MGAS2221 had ITPs, we performed Western immunoblots assessing reactivity of SpeA, SpeB, SLO, Streptococcus pyogenes NAD-glycohydrolase (SPN, also known as NADase), Mac, and S. pyogenes DNase 3 (Spd3). Mucoid and nonmucoid derivatives of strain MGAS2221 had distinct protein secretion patterns characteristic of ITP and PTP strains, respectively (Figure 2A). Assays of culture supernatant SPN enzymatic activity further supported the idea that ITP GAS can be derived from PTP GAS during invasive infections (Figure S3).

Expression microarray analysis was used to determine if the mucoid and nonmucoid mouse-passaged derivatives of strain MGAS2221 had transcriptomes characteristic of ITP and PTP GAS, respectively. The transcriptomes of 18 derivatives of strain MGAS2221 and six derivatives of strain MGAS5005 grown to early exponential phase were analyzed (Figure 2B and 2C, Table S1). As expected the mucoid derivatives of PTP strain MGAS2221 clustered with the ITP strain MGAS5005, confirming a PTP to ITP transition (Figure 2B). Transcriptome differences between strain MGAS2221 and ITP mouse-passaged derivatives correlated strongly with 
those observed for clinical PTP and ITP isolates (correlation coefficient $R^{2}=0.76$; Figure S4), further confirming the ability of M1 GAS to transition from PTP to ITP during an invasive infection.

\section{Differential Virulence of ITP and PTP GAS in Two Mouse Models of Invasive Disease}

To determine whether ITP and PTP GAS differed in ability to cause invasive disease, isolates were compared in mouse models of bacteremia and soft-tissue infection. Four ITP and

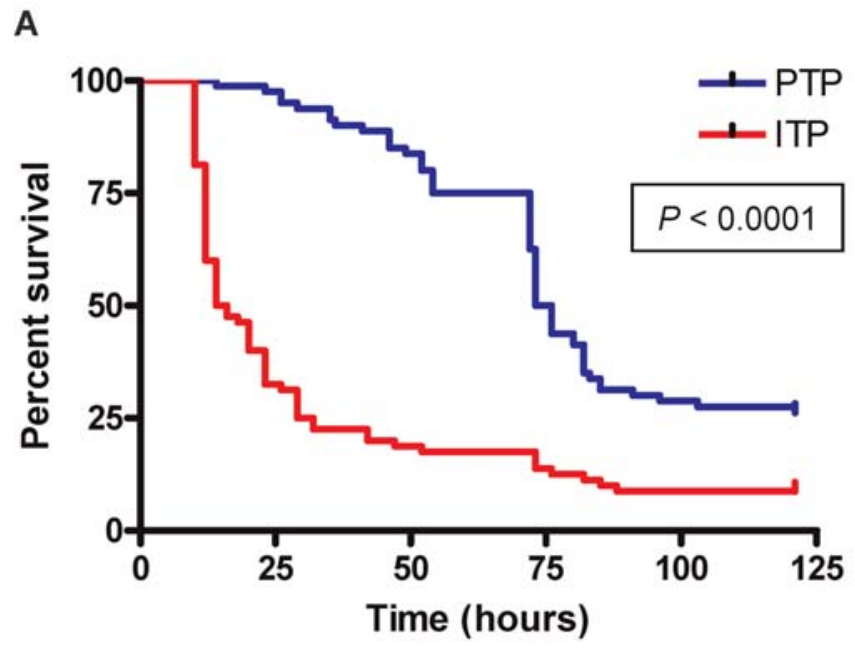

B

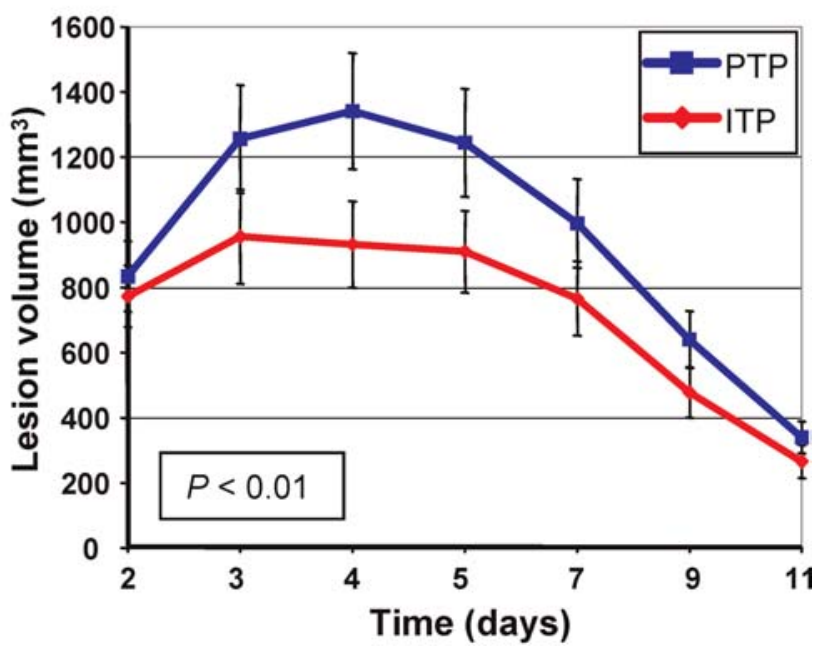

Figure 3. Differential Virulence of ITP and PTP GAS in Mouse Models of Invasive Disease

(A) ITP GAS are significantly more virulent than PTP GAS in a mouse model of bacteremia ( $p<0.0001$, logrank test). Female CD-1 mice were injected intraperitoneally with $2.5 \times 10^{7} \mathrm{CFU}$ of GAS and lethality was monitored. Four ITP and four PTP strains were each used to infect 20 mice. Survival curves were generated by pooling data from mice infected with GAS strains of the same transcriptome profile.

(B) PTP GAS produce significantly larger lesion volumes than ITP GAS in a mouse soft-tissue infection model $(p<0.01$, mixed-model repeatedmeasures analysis). Female Crl:SKH1-hrBR mice were injected subcutaneously with $1 \times 10^{7} \mathrm{CFU}$ of GAS and monitored for skin lesion formation. Shown are the average lesion volumes ( \pm standard error of the mean) of mice pooled together based on the transcriptome profile of the infecting strain. Fifteen mice were infected per GAS strain, with four ITP and four PTP strains being used.

DOI: 10.1371/journal.ppat.0020005.g003 four PTP GAS strains were randomly chosen for study. In the bacteremia model each of these eight strains was used to infect 20 CD-1 mice by intraperitoneal injection. ITP GAS were significantly more virulent than PTP GAS in this model of infection ( $p<0.0001$, logrank test; Figure 3A).

Next, the virulence of these eight ITP and PTP strains was compared in a mouse model of soft-tissue infection. Fifteen immunocompetent hairless mice were infected with each strain. The average lesion volumes over time were significantly greater for mice infected with the PTP organisms than ITP GAS (Figure 3B). Thus, in contrast to the bacteremia model data, the soft-tissue infection study identified PTP GAS as being more virulent than ITP GAS, as assessed by lesion volume. Consistent with the increased virulence of the PTP organisms during soft-tissue infection, four PTP infected animals reached near death, whereas no ITP infected mice reached this end point. Thus, it appears that the two distinct transcriptomes of GAS bestow differential virulence, with ITP GAS having increased virulence in bacteremia, and PTP GAS having increased virulence in soft-tissue infections.

Differential Resistance of ITP and PTP GAS Strains to Polymorphonuclear Leukocyte-Mediated Killing

Several of the virulence factors more highly expressed by ITP GAS compared to PTP organisms have been reported to have detrimental effects on host cells such as polymorphonuclear leukocytes (PMNs), key mediators of innate immunity. For example, streptococcal inhibitor of complement or Sic has been shown to inhibit PMN function [23]. Thus we hypothesized that ITP and PTP strains would differ significantly in ability to be killed by human PMNs. We found that ITP isolates were significantly more able to resist killing by human PMNs (Figure 4).

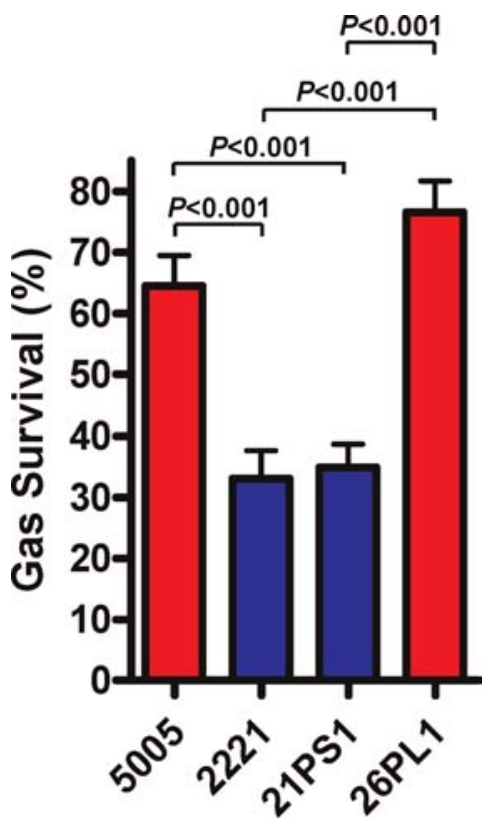

Figure 4. ITP and PTP GAS Show Differential Resistance to PMNMediated Killing

Killing of ITP GAS strains MGAS5005 and 26PL1 by human PMNs occurred at significantly lower levels than the PTP GAS strains MGAS2221 and 21PS1 ( $p<0.001$, analysis of variance with Bonferroni's posttest for multiple comparisons). Results shown are the mean \pm standard error of the mean of four experiments.

DOI: 10.1371/journal.ppat.0020005.g004 
Table 1. Genomic Differences Differentiating MGAS5005 from the Isolates MGAS2221, 26PL1, and 52SL3

\begin{tabular}{|c|c|c|c|c|c|c|}
\hline $\begin{array}{l}\text { Genome } \\
\text { Location }^{\text {a }}\end{array}$ & $\begin{array}{l}\text { DNA } \\
\text { Change }\end{array}$ & Gene & Change $^{b}$ & $\begin{array}{l}\text { MGAS } \\
2221\end{array}$ & 26PL1 & 52SL3 \\
\hline 293,185 & $+1 b p$ & covs & Wild-type & $\mathrm{Y}$ & Y & $\mathrm{N}$ \\
\hline 293,726 & $+7 \mathrm{bp}$ & covs & Frameshift & $\mathrm{N}$ & Y & $\mathrm{N}$ \\
\hline 338,178 & $A \rightarrow G$ & DNA pollII & Nonsynonymous & Y & Y & $\mathrm{N}$ \\
\hline 768,318 & $\mathrm{C} \rightarrow \mathrm{A}$ & M5005_Spy0763 & Nonsynonymous & $\mathrm{Y}$ & Y & $\mathrm{N}$ \\
\hline 782,223 & $A \rightarrow G$ & $5^{\prime}$ to $\mathrm{scl}$ & $\mathrm{n} / \mathrm{a}$ & $\mathrm{Y}$ & Y & $\mathrm{N}$ \\
\hline 791,410 & $\mathrm{~T} \rightarrow \mathrm{A}$ & Iron (III)-binding & Nonsynonymous & $\mathrm{Y}$ & Y & $\mathrm{N}$ \\
\hline 850,211 & $\mathrm{~A} \rightarrow \mathrm{T}$ & $\begin{array}{l}\text { Xanthine } \\
\text { permease }\end{array}$ & Nonsynonymous & $\mathrm{Y}$ & Y & $\mathrm{N}$ \\
\hline 957,852 & $\mathrm{~T} \rightarrow \mathrm{C}$ & M5005_Spy0967/8 & $5^{\prime}$ of genes & $\mathrm{Y}$ & $\mathrm{Y}$ & $\mathrm{N}$ \\
\hline $1,046,961$ & $\mathrm{~T} \rightarrow \mathrm{C}$ & $d l t A$ & Nonsynonymous & $\mathrm{Y}$ & Y & $\mathrm{N}$ \\
\hline $1,219,754$ & $\mathrm{~T} \rightarrow \mathrm{C}$ & Ribose repressor & Nonsynonymous & $\mathrm{Y}$ & $\mathrm{Y}$ & $\mathrm{N}$ \\
\hline $1,233,518$ & $A \rightarrow G$ & M5005_Spy1279 & Nonsynonymous & $\mathrm{Y}$ & Y & $\mathrm{N}$ \\
\hline $1,391,298$ & $A \rightarrow C$ & Phage tail & Synonymous & $\mathrm{Y}$ & $\mathrm{Y}$ & $\mathrm{N}$ \\
\hline $1,458,313$ & $A \rightarrow G$ & $\begin{array}{l}\text { P-glycerate } \\
\text { mutase }\end{array}$ & Nonsynonymous & $\mathrm{Y}$ & Y & $\mathrm{N}$ \\
\hline $1,467,369$ & $\mathrm{~T} \rightarrow \mathrm{C}$ & Stress response & Nonsynonymous & $\mathrm{Y}$ & $Y$ & $\mathrm{~N}$ \\
\hline $1,488,155$ & $A \rightarrow G$ & $\mathrm{Fe}^{+}$siderophore & Synonymous & Y & Y & $\mathrm{N}$ \\
\hline $1,624,686$ & $A \rightarrow G$ & Oxidoreductase & Synonymous & $\mathrm{Y}$ & Y & $\mathrm{N}$ \\
\hline $1,636,815$ & $A \rightarrow G$ & Pullulanase & Synonymous & Y & Y & $\mathrm{N}$ \\
\hline $1,726,511$ & $\Delta$ & IS element & Not present & $Y$ & $Y$ & $\mathrm{~N}$ \\
\hline $1,732,435$ & $\mathrm{C} \rightarrow \mathrm{T}$ & $\begin{array}{l}\text { Urocanate } \\
\text { hydratase }\end{array}$ & Synonymous & Y & Y & $\mathrm{N}$ \\
\hline $1,749,727$ & $\mathrm{~T} \rightarrow \mathrm{C}$ & Peptidase & Synonymous & $\mathrm{Y}$ & Y & $\mathrm{N}$ \\
\hline $1,809,719$ & $\mathrm{~T} \rightarrow \mathrm{C}$ & $\begin{array}{l}\text { Serine } \\
\text { dehydratase }\end{array}$ & Synonymous & Y & Y & $\mathrm{N}$ \\
\hline
\end{tabular}

$5^{\prime}$ of genes refers to SNP located upstream of genes.

'Location in MGAS5005 genome.

${ }^{b}$ Change relative to MGAS5005 sequence.

n/a, not applicable.

DOI: $10.1371 /$ journal.ppat.0020005.t001

Implication of the Role of covR/S Mutation As a Molecular Event Underpinning the PTP to ITP Transition

To test the hypothesis that the PTP to ITP transition is associated with specific chromosomal mutations, microarraybased sequencing (Nimblegen Systems) was used to compare the entire genome of one PTP and three ITP isolates. As expected, the genome sequence of strain MGAS5005 (ITP) was identical to strain 52SL3, a mouse-passaged derivative of strain MGAS5005 that also has an ITP (Table 1). The PTP strain MGAS2221 and its mouse-passaged ITP derivative strain 26PL1 had 20 common genetic changes relative to the genome of strain MGAS5005 (Table 1). More important, relative to parental strain MGAS2221 (PTP), strain 26PL1 (ITP) had only one genetic change. The unique mutation consisted of a 7-bp insertion in covS, the gene encoding the histidine kinase component of the negative regulatory TCS CovRS. This frameshift mutation results in production of a 202-amino acid truncated CovS (wild-type CovS is 500 amino acids in length). Of note, the ITP isolate MGAS5005 also has a variant $\operatorname{cov} S$ allele that truncates CovS. Taken together, these observations provide strong support to the idea that mutation of $\operatorname{cow} S$ was responsible for the PTP to ITP transition occurring during invasive infections and the accompanying differential virulence characteristics.

To further examine the association of $\operatorname{cov} R / S$ mutations with transcriptome profile, we sequenced these two genes from 42 M1 GAS isolates (15 clinical and 27 mouse-passaged derivatives of strains MGAS5005 and MGAS2221). All PTP isolates had wild-type $\operatorname{cov} R / S$ genes. In striking contrast, all ITP isolates had either a mutated $\operatorname{cov} R$ or $\operatorname{cov} S$ gene (Table S1). Thus, there was perfect correlation between the allelic state of $\operatorname{cov} R / S$ and the transcriptome profile.

\section{Complementation of the Variant covS Allele Found in Strain MGAS5005 Results in Transition from ITP to PTP}

The results above suggested that mutation of $\operatorname{cov} R$ or $\operatorname{cov} S$ was the molecular mechanism responsible for PTP to ITP transition in GAS. To further study the relationship between $\operatorname{cov} R / S$ alleles and transcriptome profile, the $\operatorname{cov} S$ variant allele in strain MGAS5005 was complemented with a functional CovS encoded by a plasmid. Transformation of strain MGAS5005 with plasmid pDC123 did not affect the secreted protein pattern, as determined by Western immunoblots assaying SpeA, SpeB, SLO/SPN, Mac, and Spd3 reactivity (Figure 5). In contrast, introduction of pDC123 encoding CovS into strain MGAS5005 converted the secretion pattern to one that was identical to PTP GAS isolates. Moreover, curing of this plasmid from the complemented strain of
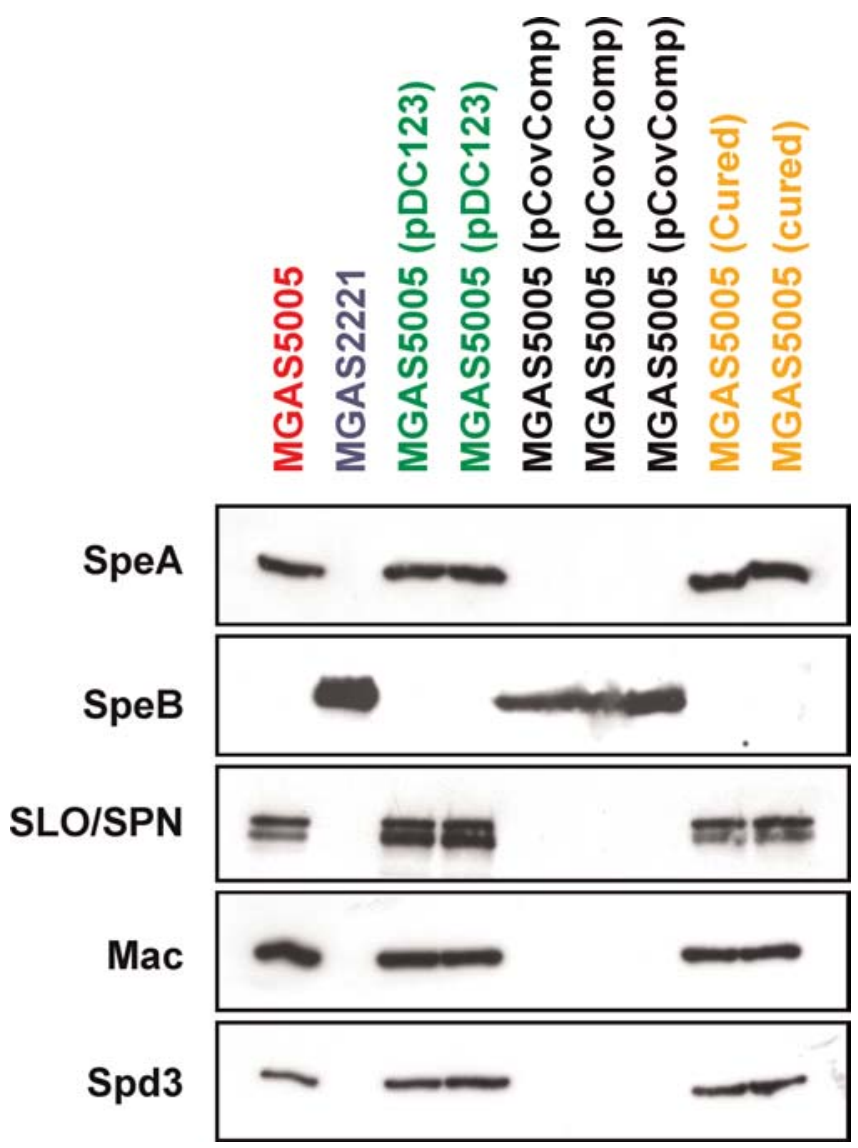

Figure 5. The ITP of MGAS5005 Is Due to a Variant covS Allele

Strain MGAS5005 was transformed with a derivative of plasmid pDC123 encoding a functional covs allele (pCovComp), and the supernatants from overnight cultures were analyzed by Western immunoblot for SpeA, SpeB, SLO/SPN, Mac, and Spd3 reactivity. Strain MGAS5005 transformed with vector $\mathrm{pDC} 123$ retained the pattern of protein secretion identified for MGAS5005 alone. In contrast, strain MGAS5005 transformed with pCovComp had an altered exoprotein secretion pattern, which was identical to that observed for PTP GAS (e.g., strain MGAS2221). Curing plasmid pCovComp from complemented MGAS5005 returned the secretion pattern to an ITP form.

DOI: 10.1371/journal.ppat.0020005.g005 
MGAS5005 produced derivatives that regained the original MGAS5005 exoprotein profile (Figure 5). Full (strain MGAS6184) or partial (strain MGAS294) complementation was also observed following introduction of the CovS encoding plasmid into two additional clinical ITP GAS strains (unpublished data). These data provide strong evidence that the transcriptome profile of GAS is intimately linked to the allelic state of $\operatorname{cov} R / S$.

\section{Discussion}

Passage of GAS in human blood ex vivo or through mice has long been known to produce strain derivatives with enhanced virulence. This strain variation also is observed among GAS clinical isolates, thereby raising the possibility that such variation is an important contributor to disease outcome. We used expression microarray and genome resequencing to characterize transcriptome differences in GAS variants and identify the underlying molecular mechanism. We discovered that a single 7-bp insertion mutation in the gene encoding the sensor kinase component of a TCS was solely responsible for the tremendous number of transcript differences distinguishing a PTP isolate from its ITP derivative.

ITP and PTP GAS strains have very distinct transcriptomes and cause significantly different disease manifestations (Figures 1 and 3). The increased virulence of ITP GAS in the bacteremia model was linked to up-regulation of many factors that inhibit PMN function, including capsule, Sic, Mac, streptococcal DNase D2, C5a peptidase, S. pyogenes cellenvelope protease, and other exoproteins that assist pathogen survival (Figures $1 \mathrm{~B}$ and $3 \mathrm{~A}$ ) [23-27]. Increased production of these virulence factors by ITP GAS occurs concurrently with decreased production of SpeB, a broadspectrum cysteine protease virulence factor (Figure 2A [2830]). SpeB degrades fibronectin, vitronectin, preinterleukin$1 \mathrm{~B}$, and other host molecules [29,31]. More important, SpeB also cleaves and inactivates bacterial proteins that contribute to host-pathogen interactions, including many cell-wall anchored and secreted GAS virulence factors such as $M$ protein [32]. Hence, despite the modest (1.16-fold) increase in emm transcription in ITP strains (Figure S1), the relative amount of mature $\mathrm{M}$ protein may be significantly greater for ITP strains because of the SpeB-mediated degradation of $\mathrm{M}$ protein by PTP GAS. Thus, decreased SpeB production may play a key role in the increased lethality of ITP GAS by preserving GAS virulence factors [30,32,33]. Indeed, an inverse correlation between in vitro $\mathrm{SpeB}$ expression and human disease severity has been observed for contemporary M1 GAS [9]. However, the in vivo consequences of this in vitro observation are unclear. Indeed, despite minimal in vitro SpeB expression by the highly virulent ITP strain MGAS5005, this strain produces extensive amounts of SpeB when grown ex vivo in human saliva [34].

Mice infected subcutaneously with PTP GAS had significantly larger skin lesions than animals injected with ITP strains (all of which were $\operatorname{cov} S^{-}$), an unexpected finding given a previous report of increased lesion formation by a $\operatorname{cov} S$ mutant strain (Figure 3B [21]). Differences between the animal studies reported by Engleberg et al. [21] and our own include their use of Cytodex beads, a different inoculum concentration, and smaller group sizes $(n=5$ mice). It is possible that the 2-fold increase in transcription of the sag operon (encoding streptolysin S) by PTP isolates is one contributory factor for the larger lesion volumes observed following PTP GAS infection (Figures $1 \mathrm{~B}$ and 3B). Streptolysin $\mathrm{S}$ is known to cause epithelial cell cytotoxicity, impede phagocytic clearance of GAS, and promote tissue necrosis $[35,36]$. It should be noted, however, that no difference was observed between four ITP and four PTP GAS strains following determination of hemolytic zone size after plating on blood agar plates (unpublished data).

Transcription of the genes encoding streptokinase (ska), Mac (mac), and capsule production (the has operon) are repressed by CovRS [16-18,25], providing an explanation for the increased expression of these genes by ITP GAS strains. While CovRS represses the has operon directly, it is not known whether repression of $s k a$ and mac is direct or indirect. Recently, CovR has been shown to directly bind and repress expression from the sagA promoter in vitro [37]. This observation differs from that observed in the present study where mutation of $\operatorname{cov} R$ or $\operatorname{cov} S$ resulted in a decrease in $\operatorname{sag} A$ transcription (Figure 1B and 2C). In addition to encoding streptolysin S, a noncoding RNA with gene regulatory properties is also transcribed from the sag locus (also referred to as pel for pleiotropic effect locus) [38]. This regulatory RNA positively regulates the expression of several GAS virulence factor-encoding genes including sic, nga, emm, and ska. As sagA transcription is increased in PTP GAS strains this would be expected to lead to increased transcription of sic, nga, emm, and ska. However, transcription of these genes is decreased in comparison to ITP GAS (Figure 1B). We believe that this observation highlights the hierarchy and extensive crosstalking that occurs between GAS transcriptional regulators. That is, we believe that despite a greater concentration of $\operatorname{sag} A$ RNA in PTP GAS strains $\left(\operatorname{sag} A^{++}, \operatorname{cov} R / S^{+}\right)$, the transcription of sic, nga, and ska are repressed compared to ITP GAS $\left(\operatorname{sag} A^{+}, \operatorname{cov} R / S^{-}\right.$) due to CovRS-mediated repression.

In comparison to the $\operatorname{sag} A$ data, production of the transcription factor-encoding gene $r g g$ correlates well with differences in known Rgg-regulated genes. Rgg acts as both a positive (e.g., speB, grab) and negative (e.g., slo, mac) regulator of transcription [39]. As $\mathrm{rgg}$ transcription is 3.8-fold higher in PTP GAS, the increase in $s p e B$ and grab transcription and decrease in slo and mac transcription by PTP GAS isolates may at least be partially mediated through greater Rgg activity.

Genome resequencing found that only a single 7-bp insertion mutation in covS differentiated PTP strain MGAS2221 from its ITP derivative (strain 26PL1), an unexpected finding given the extensive differences in transcriptome, exoproteome, and mouse virulence between these strains. Although mutations in $\operatorname{cov} R / S$ have been reported to arise during mouse passage resulting in enhanced virulence [21], the extensive effect of a $\operatorname{cow} S$ mutation on the transcriptome and resulting phenotypes was not known until the studies reported herein. While we do not believe it to be the case, the possibility that the genome resequencing study failed to identify all genomic differences between strains cannot be discounted. Documented limitations of the resequencing array approach are difficulties in identifying DNA insertions, DNA inversions, and expanded/retracted simple sequence repeats. However, our resequencing arrays identified the 1and 7-bp insertions in $\operatorname{cov} S$, and the deletion of an insertion sequence, upon confirmation of putative polymorphisms by 
PCR and sequencing (Table 1). Thus, the resequencing microarrays used in this study were powerful tools to identify genetic differences between GAS strains at a whole genome level. Even if genomic alterations were missed in our study, the strong correlation between GAS transcriptional profile and $\operatorname{cov} R / S$ genotype, coupled with $\operatorname{cov} R / S$ complementation data, strongly supports the notion that mutation of $\operatorname{cov} R / S$ is sufficient for transition from PTP to ITP.

We hypothesize that PTP GAS are preferentially suited for survival in the human pharynx. Consistent with this hypothesis are recent reports that $\mathrm{SpeB}$ is required for growth and persistence in saliva and protection from killing by LL-37, an antimicrobial compound present in high levels in human airway-surface fluid [34,40]. Nyberg et al. [40] have reported that binding of SpeB to the GAS cell surface protects the pathogen from killing by LL-37 by targeted degradation of this antibacterial factor. In this regard, we note that the gene encoding GRAB, a GAS cell-surface protein that tethers $\alpha$-2macroglobulin to the bacterial cell surface, is itself upregulated approximately 8-fold in PTP isolates (Figure 1B [41]). The $\alpha$-2-macroglobulin is a protease inhibitor that binds and retains $\mathrm{SpeB}$ at the bacterial cell surface, protecting against LL-37-mediated killing [40,41]. Thus, coordinated upregulation of SpeB and GRAB likely enhance survival of GAS in the upper respiratory tract.

ITP GAS arise from PTP precursors during invasive infections (Figures 2 and S2), presumably due in part to their increased ability to escape PMN-mediated killing (Figure 4). Since several of the antiphagocytic effectors made by ITP strains are freely diffusible, PTP GAS present at the infection site with ITP organisms might profit from a "bystander" effect. Together, these processes increase the likelihood that the infecting bacteria are able to disseminate to a new host.

We have not obtained evidence that ITP GAS can convert to a PTP form. Similarly, we did not recover PTP GAS from the upper respiratory tract of nonhuman primates with experimental GAS pharyngitis induced by an ITP strain [26] (unpublished data). These observations, coupled with the fact that many distinct $\operatorname{cov} R / S$ mutations were found in different ITP isolates, lead us to conclude that the PTP to ITP conversion is largely unidirectional. However, we cannot rule out the possibility that transition of ITP to PTP GAS occurs at a very low frequency due to back or second-site mutations.

Following the assumption that infecting GAS is a homogeneous population, clinical diagnostic laboratories generally retain only one or a small number of colonies from each infected patient. However, the data presented herein found that both ITP and PTP GAS can be recovered from an invasive infection (Figure S2). Thus, this observation may help to explain the imperfect correlations between GAS phenotypic variation and disease source seen in this and other studies (Table S1). Isolation of PTP GAS from an invasive infection does not preclude ITP GAS from also playing an important role.

\section{Concluding Comment}

Our genome-wide analysis has provided a precise explanation for global modulation of phenotype and disease specificity in a bacterial pathogen. A key discovery was the extensive differential expression of known and putative virulence factors between invasive and pharyngeal GAS isolates. This finding provides new avenues for pathogenesis studies and vaccine and therapeutics research. Inasmuch as virtually all microbes undergo phenotypic variation, it is reasonable to speculate that the genome-wide strategies used herein will be fruitful in the study of other pathogens.

\section{Materials and Methods}

Bacterial strains. The serotype M1 GAS isolates studied are listed in Table S1. All MGAS (Musser group A Streptococcus) strains were isolated from clinical sources, whereas non-MGAS strains were derived from either strain MGAS5005 (isolate names starting with "5") or MGAS2221 (isolate names starting with " 2 ") inoculated subcutaneously in mice (see below). The whole genome sequence of strain MGAS5005 has recently been determined (see Accession Numbers) [10]. The clinical isolates examined include organisms obtained in comprehensive population-based studies conducted over many years in the United States, Canada, and Finland [10]. The strains studied were not subject to systematic sampling bias, and they provide a temporally and spatially diverse collection of isolates.

Expression microarray analysis. A recently described custom-made Affymetrix chip (Affymetrix, Santa Clara, California, United States) was used for expression microarray studies [10,19]. The chip consisted of an antisense oligonucleotide array (18 $\mu \mathrm{m}$ in size) representing greater than 400,000 25-mer probes (16 pairs per probe set). It contained probe sets (42,351 features) for 2,662 predicted GAS ORFs representing a composite superset of six GAS genome sequences (M1, M3, M5, M12, M18, and M49). The chip also contained 1,925 redundant probe sets that together represent greater than $95 \%$ of the nonredundant predicted coding regions in the genome of strain SF370.

GAS strains were grown overnight at $37{ }^{\circ} \mathrm{C}\left(5 \% \mathrm{CO}_{2}\right)$ in THY broth. For the study comparing clinical isolates of GAS (Figure 1), duplicate cultures of each strain were made by diluting (1:100) overnight cultures into fresh prewarmed THY broth. For the study comparing mouse-passaged derivatives of strains MGAS5005 and MGAS2221 (Figure 2), single cultures were seeded from overnight cultures, with the exception of strains MGAS5005 and MGAS2221 for which triplicate cultures were seeded. Bacteria were grown at $37^{\circ} \mathrm{C}$ $\left(5 \% \mathrm{CO}_{2}\right)$, and RNA was obtained in early exponential phase $\left(\mathrm{OD}_{600}=\right.$ $0.14)$. RNA isolation, cDNA synthesis, labeling, and hybridization were performed as described recently [10,19]. Gene expression estimates were calculated using GCOS software v1.1.1 (Affymetrix). Data were normalized across samples to minimize discrepancies that can arise due to experimental variables (e.g., probe preparation, hybridization). A two-sample $t$-test (unequal variance) was applied to the data using the statistical package Partek Pro v5.1 (Partek, St. Louis, Missouri, United States), followed by a false discovery rate correction $(Q<0.05)$ to account for multiple testing.

Isolation of mouse-passaged GAS. Three groups of eight female Crl:SKH10hrBR mice were injected subcutaneously with $100 \mu$ l of $1 \times$ $10^{7} \mathrm{CFU}$ of strain MGAS5005 in PBS, $1 \times 10^{7} \mathrm{CFU}$ of strain MGAS2221 in PBS, or sterile PBS. The mice were euthanized $5 \mathrm{~d}$ after injection. The spleens and skin lesions were harvested, macerated in $500 \mu \mathrm{l} \mathrm{PBS}$, and cultured overnight on blood agar plates. Single colonies were picked, grown in $1 \mathrm{ml}$ of THY broth for $7 \mathrm{~h}$, and frozen in liquid nitrogen after the addition of glycerol (final concentration, $18 \%)$.

Western immunoblot analysis of culture supernatant proteins. GAS were grown in THY broth $(10 \mathrm{ml})$ to stationary phase (overnight), and culture supernatants were obtained by centrifugation and filtration through a $0.22-\mu \mathrm{m}$ filter. Proteins were precipitated by the addition of 3.5 volumes ice-cold ethanol, collected by centrifugation, and suspended in $500 \mu \mathrm{l}$ of water. The anti-SLO antibody is known to cross-react with both SLO and SPN because of the physical association of these proteins in the immunizing antigen preparation [10].

NADase enzyme activity assay. Secreted NADase activity was assayed as described previously [10]. Briefly, supernatants from overnight cultures were centrifuged, filtered, and 2-fold serial dilutions were made in PBS. NAD ${ }^{+}$(Sigma, St. Louis, Missouri, United States), diluted in PBS, was added to a concentration of 0.67 $\mathrm{mM}$, and the reaction mixture was incubated for $1 \mathrm{~h}$ at $37^{\circ} \mathrm{C}$. $\mathrm{NaOH}$ was added to $2 \mathrm{~N}$, incubated for $1 \mathrm{~h}$ in the dark, and read by eye after exciting the samples with 360-nm light. Results were reported as the greatest 2-fold dilution of supernatant with no fluorescence.

Mouse infection assays. Mice were infected with GAS grown to midlogarithmic phase $\left(\mathrm{OD}_{600}\right.$ approximately 0.55). ITP GAS strains used in mice assays were MGAS294, MGAS5005, MGAS6184, and 
26PL1. PTP GAS strains used in mice assays were MGAS2221, MGAS5456, MGAS9127, and 21PS1. For intraperitoneal injection, 20 female CD-1 mice were used for each GAS strain. The animals were injected with $250 \mu \mathrm{l}$ of a $1 \times 10^{8} \mathrm{CFU} / \mathrm{ml}$ suspension of GAS in PBS. Kaplan-Meier survival curves were generated and analyzed for statistical significance using the logrank test. For subcutaneous inoculation, 15 female Crl:SKH10hrBR mice were injected with 100 $\mu \mathrm{l}$ of a $1 \times 10^{8} \mathrm{CFU} / \mathrm{ml}$ suspension of GAS in PBS. Lesion areas and volumes were determined daily for up to $2 \mathrm{wk}$ as previously described [26]. A mixed-model repeated-measures analysis was used to test for significant differences in lesion areas and volumes between GAS strains. The repeated-measures model was employed with one withinsubjects and one between-subjects factor.

PMN-mediated killing assays. Killing of GAS by human PMNs was determined as described previously [42], but with slight modification. In brief, PMNs $\left(10^{6}\right)$ were combined with $10^{7}$ unopsonized GAS in 96well plates on ice. Plates were centrifuged at $380 \times g$ for $5 \mathrm{~min}$ and incubated at $37^{\circ} \mathrm{C}$ for $60 \mathrm{~min}$. PMNs were lysed with $0.1 \%$ saponin (20 min on ice), and GAS were plated on THY or blood agar. Colonies were counted and percentage GAS survival was calculated with the equation

$$
\left(\mathrm{CFU}_{\mathrm{PMN}+} / \mathrm{CFU}_{\mathrm{PMN}-}\right) \times 100
$$

The assay measures total number of viable ingested and uningested bacteria.

Microarray-based comparative genome resequencing. Genome resequencing was performed by an array-based method (NimbleGen Systems, Madison, Wisconsin, United States). The genome sequence of serotype M1 strain MGAS5005 was used as the reference sequence [10]. PCR and sequencing were used to verify all polymorphisms (see Protocol S1).

Complementation of the $\operatorname{cov} S$ mutation of strain MGAS5005. The Escherichia coli-GAS shuttle vector pDC123 was used for complementation. The wild-type $\operatorname{cov} R / S$ alleles from MGAS2221 were amplified by PCR using primers COVRSF ( $5^{\prime}$-gcttgcaagggttgtttgatg- $\left.3^{\prime}\right)$ and COVRSR (5'-gcggatccttaagctactctaactctc- $\left.3^{\prime}\right)$. Purified PCR product was blunt ended, using the Klenow fragment of DNA PolI (New England Biolabs, Beverly, Massachusetts, United States), and digested with BamHI, which cuts within the site engineered into the COVRSR primer (italicized). The PCR product was ligated into pDC123 after digestion with BgIII EcoRV, to create pCovComp, and the ligation mixture was transformed directly into competent cells of strain MGAS5005 by electroporation. The vector only also was transformed into strain MGAS5005 to serve as a negative control. Transformation mixtures were plated onto THY agar plates containing $4 \mu \mathrm{g} / \mathrm{ml}$ chloramphenicol and incubated overnight at $37{ }^{\circ} \mathrm{C}\left(5 \% \mathrm{CO}_{2}\right)$. The chromosomal and plasmid covR/S genes of transformants were verified by PCR and sequencing.

\section{Supporting Information}

Figure S1. Global Transcriptome Variation between Clinical ITP and PTP GAS Strains

The $\mathrm{x}$-axis represents the whole genome sequence of contemporary M1 GAS strain MGAS5005. The $y$-axis represents the mean relative increase or decrease in gene transcription for the three clinical ITP strains in relation to that observed for the six clinical PTP strains. The dashed green lines represent \pm 2 -fold transcriptional difference. Virulence factors/regulators transcribed $>2$-fold by ITP strains are colored red. Virulence factors/regulators transcribed $>2$-fold by PTP

\section{References}

1. van der Woude MW, Baumler AJ (2004) Phase and antigenic variation in bacteria. Clin Microbiol Rev 17: 581-611.

2. Cunningham MW (2000) Pathogenesis of group A streptococcal infections Clin Microbiol Rev 13: 470-511.

3. Kotb M, Norrby-Teglund A, McGeer A, El-Sherbini H, Dorak MT, et al. (2002) An immunogenetic and molecular basis for differences in outcomes of invasive group A streptococcal infections. Nat Med 8: 1398-1404.

4. Dochez AR, Avery OT, Lancefield RC (1919) Studies on the biology of Streptococcus: I. Antigenic relationships between strains of Streptococcus hemolyticus. J Exp Med 30: 179-213.

5. Todd EW, Lancefield RC (1928) Variants of hemolytic streptococci: Their relation to type-specific substance, virulence, and toxin. J Exp Med 48: 751-767.

6. Rothbard S, Watson RF (1948) Variation occurring in group A streptococci during human infection. J Exp Med 87: 521-533.

7. Molinari G, Chhatwal GS (1998) Invasion and survival of Streptococcus strains are colored blue. The emm gene, encoding the important virulence factor $\mathrm{M}$ protein, is highlighted yellow for reference. Found at DOI: 10.1371/journal.ppat.0020005.sg001 (155 KB PPT).

Figure S2. Schematic of Experiment Leading to Isolation of MousePassaged GAS Derivatives

PTP GAS (blue box, nonmucoid) or ITP GAS (red box, mucoid) were injected subcutaneously into mice. Five days after infection mice were euthanized and GAS isolated from spleens and skin lesions. ITP GAS were isolated from the spleens and skin lesions of all infected mice. GAS recovered from skin lesions of mice infected with PTP GAS had an approximately 1:1 ratio of ITP to PTP GAS.

Found at DOI: 10.1371/journal.ppat.0020005.sg002 (9.2 MB PPT).

Figure S3. ITP Strains Secrete Increased NADase Activity Compared to PTP Strains

NADase titers are shown on the y-axis, with different GAS strains shown on the $\mathrm{x}$-axis. Color coding is as described for Figure 2B. The experiment was performed in duplicate and results identical to those shown were obtained on both occasions. NEG, negative controls.

Found at DOI: 10.1371/journal.ppat.0020005.sg003 (29 KB PPT).

Figure S4. Correlation of Microarray Data between ITP/PTP GAS Isolated from Clinical Sources and following Mouse Passage

The fold change in transcript levels (ITP relative to PTP) of 24 virulence-related genes from the clinical GAS microarray (Figure 1) and the mouse-passaged GAS microarray (Figure 2) were logtransformed and plotted against each other to evaluate their correlation.

Found at DOI: 10.1371/journal.ppat.0020005.sg004 (44 KB PPT).

Protocol S1. Comparative Genomic Resequencing

Found at DOI: 10.1371/journal.ppat.0020005.sd001 (27 KB DOC).

Table S1. Serotype M1 Group A Streptococcus Isolates Studied Found at DOI: 10.1371/journal.ppat.0020005.st001 (95 KB DOC).

\section{Accession Numbers}

Expression microarray data have been deposited at the Gene Expression Omnibus database at National Center for Biotechnology Information (http://www.ncbi.nlm.nih.gov/geo) and are accessible through accession numbers GSE3899 and GSE3900.

The GenBank (http://www.ncbi.nlm.nih.gov) accession number for the whole genome sequence of strain MGAS5005 is CP000017.

\section{Acknowledgments}

We thank M. Nagiec, D. Welty, and D. Long for assistance with animal studies. This study was supported in part by National Institutes of Health grant AI-UO1-060595 to JMM.

Author contributions. PS, FRD, and JMM conceived and designed the experiments. PS and ARW performed the experiments. PS, ARW, EAG, FRD, and JMM analyzed the data. PS and JMM wrote the paper.

Competing interests. The authors have declared that no competing interests exist.

pyogenes in eukaryotic cells correlates with the source of the clinical isolates. J Infect Dis 177: 1600-1607.

8. Shiseki M, Miwa K, Nemoto Y, Kato H, Suzuki J, et al. (1999) Comparison of pathogenic factors expressed by group A streptococci isolated from patients with streptococcal toxic shock syndrome and scarlet fever. Microb Pathog 27: 243-252.

9. Kansal RG, McGeer A, Low DE, Norrby-Teglund A, Kotb M (2000) Inverse relation between disease severity and expression of the streptococcal cysteine protease, SpeB, among clonal M1T1 isolates recovered from invasive group A streptococcal infection cases. Infect Immun 68: 6362-6369.

10. Sumby P, Porcella SF, Madrigal AG, Barbian KD, Virtaneva K, et al. (2005) Evolution and emergence of a highly successful clone of serotype M1 group A Streptococcus involved multiple horizontal gene transfer events. J Infect Dis 192: 771-782.

11. Raeder R, Harokopakis E, Hollingshead S, Boyle MD (2000) Absence of SpeB production in virulent large capsular forms of group A streptococcal strain 64. Infect Immun 68: 744-751. 
12. Kazmi SU, Kansal R, Aziz RK, Hooshdaran M, Norrby-Teglund A, et al. (2001) Reciprocal, temporal expression of SpeA and SpeB by invasive M1T1 group A streptococcal isolates in vivo. Infect Immun 69: 4988-4995.

13. Smith TC, Sledjeski DD, Boyle MD (2003) Streptococcus pyogenes infection in mouse skin leads to a time-dependent up-regulation of protein $\mathrm{H}$ expression. Infect Immun 71: 6079-6082.

14. Rezcallah MS, Boyle MD, Sledjeski DD (2004) Mouse skin passage of Streptococcus pyogenes results in increased streptokinase expression and activity. Microbiology 150: 365-371.

15. Kreikemeyer B, McIver KS, Podbielski A (2003) Virulence factor regulation and regulatory networks in Streptococcus pyogenes and their impact on pathogen-host interactions. Trends Microbiol 11: 224-232.

16. Levin JC, Wessels MR (1998) Identification of $c s r R / c s r S$, a genetic locus that regulates hyaluronic acid capsule synthesis in group A Streptococcus. Mol Microbiol 30: 209-219.

17. Federle MJ, McIver KS, Scott JR (1999) A response regulator that represses transcription of several virulence operons in the group A Streptococcus. J Bacteriol 181: 3649-3657.

18. Graham MR, Smoot LM, Migliaccio CA, Virtaneva K, Sturdevant DE, et al. (2002) Virulence control in group A Streptococcus by a two-component gene regulatory system: Global expression profiling and in vivo infection modeling. Proc Natl Acad Sci U S A 99: 13855-13860.

19. Graham MR, Virtaneva K, Porcella SF, Barry WT, Gowen BB, et al. (2005) Group A Streptococcus transcriptome dynamics during growth in human blood reveals bacterial adaptive and survival strategies. Am J Pathol 166: 455-465.

20. Gryllos I, Levin JC, Wessels MR (2003) The CsrR/CsrS two-component system of group A Streptococcus responds to environmental Mg2+. Proc Natl Acad Sci U S A 100: 4227-4232.

21. Engleberg NC, Heath A, Miller A, Rivera C, DiRita VJ (2001) Spontaneous mutations in the CsrRS two-component regulatory system of Streptococcus pyogenes result in enhanced virulence in a murine model of skin and soft tissue infection. J Infect Dis 183: 1043-1054.

22. Musser JM, DeLeo FR (2005) Toward a genome-wide systems biology analysis of host-pathogen interactions in group A Streptococcus. Am J Pathol 167: $1461-1472$.

23. Hoe NP, Ireland RM, DeLeo FR, Gowen BB, Dorward DW, et al. (2002) Insight into the molecular basis of pathogen abundance: Group A Streptococcus inhibitor of complement inhibits bacterial adherence and internalization into human cells. Proc Natl Acad Sci U S A 99: 7646-7651.

24. Ji Y, McLandsborough L, Kondagunta A, Cleary PP (1996) C5a peptidase alters clearance and trafficking of group A streptococci by infected mice. Infect Immun 64: 503-510.

25. Lei B, DeLeo FR, Hoe NP, Graham MR, Mackie SM, et al. (2001) Evasion of human innate and acquired immunity by a bacterial homolog of CD11b that inhibits opsonophagocytosis. Nat Med 7: 1298-1305.

26. Sumby P, Barbian KD, Gardner DJ, Whitney AR, Welty DM, et al. (2005) Extracellular deoxyribonuclease made by group A Streptococcus assists pathogenesis by enhancing evasion of the innate immune response. Proc Natl Acad Sci U S A 102: 1679-1684.

27. Edwards RJ, Taylor GW, Ferguson M, Murray S, Rendell N, et al. (2005) Specific c-terminal cleavage and inactivation of interleukin- 8 by invasive disease isolates of Streptococcus pyogenes. J Infect Dis 192: 783-790.
28. Lukomski S, Burns EH Jr, Wyde PR, Podbielski A, Rurangirwa J, et al. (1998) Genetic inactivation of an extracellular cysteine protease (SpeB) expressed by Streptococcus pyogenes decreases resistance to phagocytosis and dissemination to organs. Infect Immun 66: 771-776.

29. Rasmussen M, Bjorck L (2002) Proteolysis and its regulation at the surface of Streptococcus pyogenes. Mol Microbiol 43: 537-544.

30. Aziz RK, Pabst MJ, Jeng A, Kansal R, Low DE, et al. (2004) Invasive M1T1 group A Streptococcus undergoes a phase-shift in vivo to prevent proteolytic degradation of multiple virulence factors by SpeB. Mol Microbiol 51: 123134.

31. Kapur V, Majesky MW, Li LL, Black RA, Musser JM (1993) Cleavage of interleukin 1 beta (IL-1 beta) precursor to produce active IL-1 beta by a conserved extracellular cysteine protease from Streptococcus pyogenes. Proc Natl Acad Sci U S A 90: 7676-7680.

32. Raeder R, Woischnik M, Podbielski A, Boyle MD (1998) A secreted streptococcal cysteine protease can cleave a surface-expressed M1 protein and alter the immunoglobulin binding properties. Res Microbiol 149: 539548.

33. Wei L, Pandiripally V, Gregory E, Clymer M, Cue D (2005) Impact of the SpeB protease on binding of the complement regulatory proteins factor $\mathrm{H}$ and factor H-like protein 1 by Streptococcus pyogenes. Infect Immun 73: 20402050 .

34. Shelburne SA III, Granville C, Tokuyama M, Sitkiewicz I, Patel P, et al. (2005) Growth characteristics of and virulence factor production by group A Streptococcus during cultivation in human saliva. Infect Immun 73: 47234731 .

35. Engleberg NC, Heath A, Vardaman K, DiRita VJ (2004) Contribution of CsrR-regulated virulence factors to the progress and outcome of murine skin infections by Streptococcus pyogenes. Infect Immun 72: 623-628.

36. Datta V, Myskowski SM, Kwinn LA, Chiem DN, Varki N, et al. (2005) Mutational analysis of the group A streptococcal operon encoding streptolysin $\mathrm{S}$ and its virulence role in invasive infection. Mol Microbiol 56: 681-695.

37. Gao J, Gusa AA, Scott JR, Churchward G (2005) Binding of the global response regulator protein CovR to the sag promoter of Streptococcus pyogenes reveals a new mode of CovR-DNA interaction. J Biol Chem 280: $38948-38956$

38. Mangold M, Siller M, Roppenser B, Vlaminckx BJ, Penfound TA, et al. (2004) Synthesis of group A streptococcal virulence factors is controlled by a regulatory RNA molecule. Mol Microbiol 53: 1515-1527.

39. Chaussee MS, Sylva GL, Sturdevant DE, Smoot LM, Graham MR, et al. (2002) Rgg influences the expression of multiple regulatory loci to coregulate virulence factor expression in Streptococcus pyogenes. Infect Immun 70: 762-770.

40. Nyberg P, Rasmussen M, Bjorck L (2004) Alpha2-macroglobulin-proteinase complexes protect Streptococcus pyogenes from killing by the antimicrobial peptide LL-37. J Biol Chem 279: 52820-52823.

41. Rasmussen M, Muller HP, Bjorck L (1999) Protein GRAB of Streptococcus pyogenes regulates proteolysis at the bacterial surface by binding alpha2macroglobulin. J Biol Chem 274: 15336-15344.

42. Kobayashi SD, Braughton KR, Whitney AR, Voyich JM, Schwan TG, et al. (2003) Bacterial pathogens modulate an apoptosis differentiation program in human neutrophils. Proc Natl Acad Sci U S A 100: 10948-10953. 